\title{
Cognitive Profiles in Patients with Multi-Infarct Dementia: An Omani Study
}

\author{
Samir Al-Adawi ${ }^{a} \quad$ Nady Braidyg Musthafa Essa $^{c}$ Faisal Al-Azrib \\ Samir Hussain ${ }^{b}$ Nasser Al-Sibani ${ }^{d}$ Jabar Al-Khabouri ${ }^{\mathrm{e}}$ \\ Abdullah Al-Asmie Ali Al-Mashani ${ }^{f}$ \\ a Department of Behavioral Medicine, College of Medicine and Health Sciences, \\ ${ }^{b}$ Department of Radiology and Molecular Imaging, Sultan Qaboos University Hospital, and \\ 'Department of Food Science and Nutrition, CAMS, Sultan Qaboos University, \\ ${ }^{\mathrm{d}}$ Al-Masara Hospital, Ministry of Health, ${ }^{e}$ Department of Neurology, Royal Hospital, \\ Ministry of Health, and ${ }^{\mathrm{f}}$ Khoula Hospital, Muscat, Oman; ${ }^{\mathrm{g} C e n t r e ~ f o r ~ H e a l t h y ~ B r a i n ~ A g e i n g, ~}$ \\ School of Psychiatry, University of New South Wales, Sydney, N.S.W., Australia
}

Key Words

Multi-infarct dementia · Cognition · Quality of sleep · Depression · Memory

\section{Abstract}

Background: Studies on neurocognitive impairment among patients presenting with multiinfarct dementia (MID) have received little attention from non-Western societies, and the Arab world is no exception. To our knowledge, this is the first study to characterize neurocognitive, affective and vegetative functioning in patients with MID in Oman. Methods: In this study, we recruited 20 Omani patients presenting with MID and age- and gender-matched controls at the outpatient clinic of the Department of Behavioral Medicine, Sultan Qaboos University Hospital, Sultan Qaboos University, Muscat, Oman. In addition to the collection of clinical and demographic information, various cognitive batteries were administered to the consenting participants, including those indexing nonverbal reasoning abilities, working memory (attention, concentration and recall) and executive functioning. Questionnaires that elicit the affective range and the quality of sleep were also administered. Results: Compared with the matched healthy subjects, the patients diagnosed with MID significantly differed in the presently operationalized indices of visuospatial function, semantic memory and affective and vegetative functioning. In contrast, episodic memory and some attentional capacities were not significantly different compared with the control subjects. Conclusions: The present study was explorative and clinically designed to describe neurocognitive functioning in patients with MID seeking consultation at a tertiary care center in Oman. Our data are necessary for planning and setting up community services and health care programs for demented patients in a society where dementia is a growing silent epidemic. 


\section{Introduction}

Alzheimer's disease (AD) and multi-infarct dementia (MID) represent the most common causes of cognitive impairment and the resultant social deficits in the elderly population [1]. While AD is more prevalent, MID nonetheless affects up to $30 \%$ of the elderly individuals over 65 years with cognitive impairment. Recent epidemiological studies have shown that the prevalence of dementia exceeds 7 million in the USA alone, with some 4.5 million of those individuals suffering from AD and 2.5 million from MID [2]. However, in some non-Western countries, there are more reported cases of cognitive impairment that owe their origin to vascular pathology than to other pathologies [3], but there is a dissenting view [4]. A similar trend is likely to exist in other countries, since increased life-span plasticity and the emergence of neurodegenerative diseases are strong predictors of cognitive impairment $[5,6]$.

MID is caused by a series of cumulative and insidious strokes that culminate in overt infarcts after a span of time. Silent strokes affect only small areas of the brain, but over time, the symptoms of MID begin to develop as more areas of the brain are affected [7]. Larger strokes which affect strength, sensation and other neurological functions can also trigger MID. As the term suggests, multi-infarct means that several areas of the brain are affected due to reduced blood flow to those regions, leading to hypoxia and culminating in neurodegeneration and permanent cell damage and the resultant cognitive impairment [8].

While the causes of dementia have traditionally been considered separately within a specific individual, recent studies have shown that patients with cerebrovascular abnormalities show a higher proclivity for developing $\mathrm{AD}$, and up to $60 \%$ of these patients also demonstrate vascular impairments which are common to MID [9-12]. The definition of dementia strictly requires the presence of significant memory impairment and delayed cognitive functioning, that is, at least 1 of the following: aphasia, apraxia, agnosia and/or impairment of executive functioning. Owing to the inherent variability in vascular changes and cognitive decline in MID, patients may demonstrate different cognitive impairment profiles and varying levels of severity [13-17]. Also, individuals with MID usually demonstrate less severe memory difficulties as compared with other cognitive limitations. Furthermore, the formal diagnosis of MID requires an association of demonstrable vascular changes with cognitive difficulties $[10-12,18]$. This may lead to an underdiagnosis of dementia and the inability to fully access all individuals with cognitive decline in a given population.

There is a dearth of studies elucidating the magnitude of neurocognitive impairment in non-Western populations [6]. Little empirical evidence is found on how individuals with mild cognitive impairment (MCI) fare with regard to indices of cognitive functioning, affective range and vegetative functioning in Arab/Islamic populations. Oman, which is located in the southern tip of the Arabian Peninsula, offers an interesting ground for exploring the applicability of the Western model of cognitive functioning, emotionality and quality of sleep. Some of the putative contributors to MID are hypertension, hyperlipidemia and diabetes. These conditions are becoming increasingly prevalent with the onset of recent affluence [19].

The current population pattern in Oman fits with a second phase of demographic transition characterized by reduced mortality rates but complemented by increased life expectancy [20]. Increased longevity is known to be the strongest risk factor for age-related dementias. Moreover, the prevalence of MID in non-Western countries such as Oman has not been formally established. Anecdotal reports point to an emergence of age-related cognitive impairment in Arab communities [21, 22]. However, the quantification of the magnitude of cognitive impairment has been hampered by the fact that most of the available screening tools are known to require arithmetic ability or reading or writing skills, which are often absent in societies where illiteracy is prevalent among the elderly population [21, 22]. A novel approach is needed to quantify the presence of cognitive disorder in non-Western populations. 
The variability in anatomic and neurological changes in MID plays a significant role in the pathological processes that directly affect neurocognitive functioning, the affective range and vegetative functioning. In this report, we assessed neurocognitive functioning in subjects with MID. MID and control patients were subjected to assessments using several behavioral batteries that are known to elicit various repertoires of cognitive functioning, emotional integrity and quality of sleep.

\section{Patients and Methods}

\section{Patients}

Oman's national health care service is free for its nationals. Tertiary care is largely compartmentalized and centralized in Oman. The study has been approved by the Medical Research Ethics Committee at the College of Medicine and Health Sciences, Sultan Qaboos University. The present cohort $(n=20)$, the consecutive attendees of 1 year (2007), was referred to the outpatient clinic of the Department of Behavioral Medicine, Sultan Qaboos University Hospital. This is the only center where the quantification of the level of a disability is possible in Oman. These outpatients were usually in the early stages of their disease and referred to the outpatient clinic for routine diagnostic evaluation. The degree of impairment was compared with that in normal age-matched healthy subjects $(n=18)$. Those included were drug-naïve consecutive patients with a large area without tracer uptake in brain regions critically associated with the pathology of dementia, quantified by single-photon emission computed tomography, as exemplified by figure 1 .

The diagnosis of MID utilized the DSM-IV criteria and was based on: (1) a history of multiple cognitive deficits; (2) cognitive deficits which can cause significant impairment of social or occupational functioning; (3) the presence of focal neurological signs and symptoms or laboratory evidence of cerebrovascular disease, such as a history of at least 1 definite stroke, or at least 1 visible cerebral lesion detected in the brain using CT scanning and an Ischemia Scale score of 7 or more, and (4) deficits not exclusive to delirium. None of the patients had visual field impairments upon physical examination. Patients with major cortical strokes or strategic thalamic infarcts were excluded from this study. We quantified dementia severity using a standardized and validated Arabic version of the Mini-Mental State Examination (MMSE). A score greater than or equal to 25 was considered cognitively normal. Patients with MID were included in this study if they scored 24 or less.

\section{Assessment Measures}

Information concerning neurocognitive, vegetative and affective functioning was obtained by interviewing the patients and, if possible, members of the their family, and by reviewing the available hospital charts. The tests used to assess the performance in each domain are described below.

Nonverbal Reasoning Ability

Raven's Progressive Matrices. Raven's Progressive Matrices (RPM) measures the current nonverbal reasoning ability [23]. It has been employed as a 'culture-free' test, since it is relatively independent of language and educational skills [24]. It consists of 60 items grouped into 5 sets. Each item contains a pattern with 1 part removed and between 6 and 8 pictured inserts, 1 of which contains the correct missing part. The subject is required to point to the pattern he/she selects as correct. The present score constitutes a raw score based on the number of correct patterns, with a maximum score of 60 . 


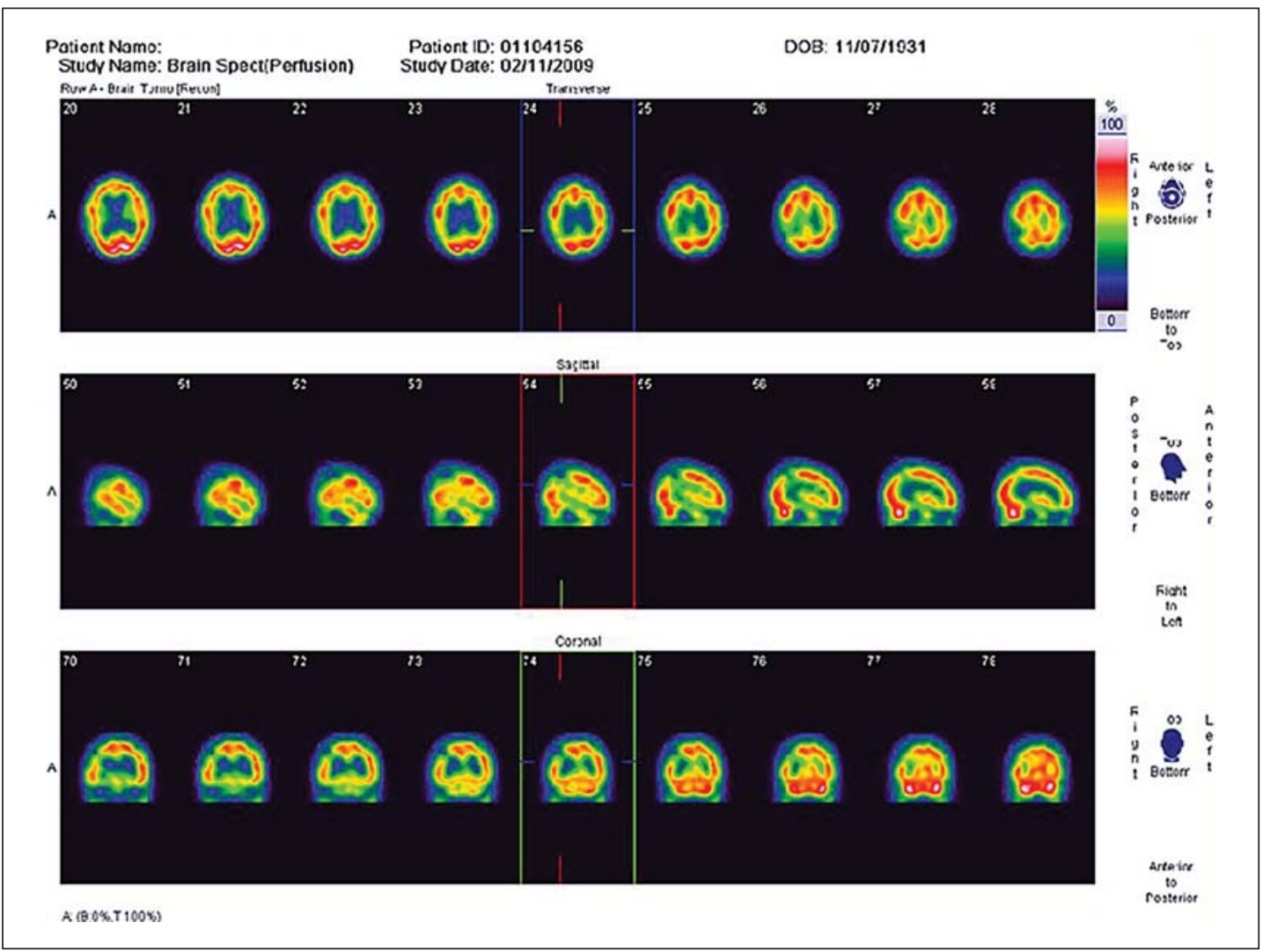

Fig. 1. Brain perfusion ( ${ }^{99 \mathrm{~m}} \mathrm{Tc}$, Neurolite; dose: $655 \mathrm{MBq}$ ) images of 1 typical patient with MID included in this study. There was a large area without tracer uptake in the right parietal lobe and another smaller area in the right temporal lobe. There were multiple other areas with reduced tracer uptake in the left frontal and parietal lobes.

\section{Working Memory}

Digit Span Test. The digit span test [25] has two components: forward and backward. In the digit span forward test, the examiner reads out a list of digits at the rate of 1 digit/s and the subject is required to repeat the sequence in the same order. The first sequence comprises 2 digits, and if the subject succeeds with this task, sequences of increasing length are presented. Two sequences of each length are given, and the test is stopped when the subject fails to repeat both sequences of a particular length. The same procedure is followed in the digit span backward test, with the difference that, in this case, subjects are requested to reproduce the sequences of digits in reverse order. In the emerging literature, the digit span test has been indicated to subserve indices of working memory [26].

Buschke Selective Reminding Test. In essence, the Buschke Selective Reminding Test (BSRT) [27] examines the rate of learning new information. The subject recalls as many words as he/she can, in any order, from a list of 12 items (of a single semantic category, e.g. animals) just read to him/her. After each recall trial, the examiner repeats only those items which the subject omitted in his/her recall. This technique has been used to provide a means of assessing retention and retrieval. It is an effortful task in that learning and recall can be enhanced by the application of encoding and/or retrieval strategies and/or by rehearsal [28]. Such cognitive operation has been widely referred to as working memory $[29,30]$ or working with memory [31]. 


\section{Executive Functioning}

Tower of London Test. The Tower of London (ToL) test is a problem-solving task composed of 3 wooden rods of differing lengths attached to a block base and 3 balls (red, green and blue) placed on the rods in a predefined position. For each activity to be completed, these 3 balls must be moved from the starting configuration to the target position with the minimum number of moves. There is evidence that this task taps visuospatial planning and problem solving, which is often viewed as an integral part of executive functioning in the parlance of neuropsychology [28]. For the present purpose, the scoring system is based on the number of correctly solved problems with a maximum correct score of 12 , as described elsewhere [32].

Verbal Fluency Test. The verbal fluency test (Controlled Oral Word Association Test) [33] examines the initiation and speed of verbal responses. Subjects are asked to produce as many different words as possible that begin with each of 3 specific letters, with $1 \mathrm{~min} /$ letter. They are explicitly told not to repeat the same word and not to say the same word with a different ending (e.g. fish, fishing, fisherman). Following instructions, examples and a demonstration so that they understand the task requirements, they are given each letter in turn. The total score for verbal fluency thus is the total number of different acceptable words produced across the three 60-second periods. In this study, the letters were taa, raa and waaw of the Arabic alphabet, as described elsewhere [34]. The neuropsychological underpinnings of verbal fluency have been linked to the integrity of the dorsolateral prefrontal cortex, which, in turn, has been associated with executive functioning [35, 36].

Wisconsin Card Sorting Test. The modified Wisconsin Card Sorting Test (WCST) [37] assesses executive function based on the principles according to which a deck of cards must be sorted [28]. The cards have geometric figures which vary simultaneously in shape (triangle, star, cross or circle), color (green, red, blue or yellow) and number (1, 2, 3 or 4 figures). Four reference cards are permanently placed in front of the subject. The subject has another deck of cards called reference or response cards. She or he is asked to match each response card successively with 1 of the 4 reference cards. The experimenter selects 1 of the dimensions (e.g. color) as the sorting rule. The first rule in fact reflects the first card placement made by the subject. After each response, she or he is told only whether it was correct or not. If the response was incorrect, she or he is not permitted to move it but is given the next card to try and place it appropriately. Normal subjects use a hypothesis-testing or deductive reasoning approach to identify which sorting rule is in operation. When she or he has correctly placed 6 consecutive cards, she or he is told simply that the rule has changed. The second rule in fact is selected according to the subjects' next card placement, which uses a different rule. After this has been correctly employed 6 times in succession, the rule changes again. The third rule is the remaining dimension (shape, color or number). The 3 rules are then cycled through again in the same order. This procedure is continued until 6 categories are achieved or the pack of 48 cards is used up. The present score is calculated according to the scoring system of Nelson [37]. Accordingly, 1 index was recorded: the number of categories achieved, as described elsewhere [28].

Trail-Making Test. As described elsewhere [32], the scoring for the Trail-Making Test was derived from two versions of the test. The first one requires the examinee to draw a line to connect, in correct order, the numbers 1 through 25 , each contained in a plain black circle. In the second version, the examinee draws a line to connect, in alternating sequence, the numbers 1 through 12 and the letters $\mathrm{A}$ through $\mathrm{L}$, beginning with 1 and drawing a line to $\mathrm{A}$, then 2 , then $B$, and so on, until all the numbers and letters are connected. For the present context, test total times for both versions of the test were scored in seconds. 
Al-Adawi et al.: Cognitive Profiles in Patients with Multi-Infarct Dementia: An Omani Study

Table 1. Demographic data and measures of the general level of cognitive decline for MID patients and controls

\begin{tabular}{lcc}
\hline & MID patients & Controls \\
\hline Number (M/F) & $20(16 / 4)$ & $18(17 / 1)$ \\
Age range (mean), years & $60-82(71)$ & $59-79(69)$ \\
Years of full-time education & $11.3 \pm 2.5$ & $11.8 \pm 2.9$ \\
MMSE score & $23.5 \pm 4.6$ & $28.2 \pm 1.5^{*}$ \\
\hline
\end{tabular}

Values are displayed as means \pm SD unless specified otherwise. The maximum score for the MMSE is 30. Descriptions of the MID and control groups are included in the text. $* p<0.05$ using Student's t test on the group means.

Affective Range

Geriatric Depression Scale. The 15-item Geriatric Depression Scale (GDS-15) [38] has been established to identify adequate psychometric properties in geographically different populations [39], including Arab-speaking ones [40]. The scale requires subjects to give their responses in reference to the previous week. One point is given for each positive response, and the number of points is summed to provide a single score. In the present context, the higher the score, the higher the propensity for depression which could be qualified as 'caseness'.

Quality of Sleep

Pittsburg Sleep Quality Index. The Pittsburg Sleep Quality Index (PQSI) is a 21-point assessment task which comprises 7 components including subjective sleep quality, sleep latency, sleep duration, sleep efficiency, sleep disturbances, the use of sleep medication and daytime dysfunction [32]. The purpose of this task is to evaluate sleep patterns during the previous month. Subjects with a high score have a poor sleep quality and suffer all the consequences this may entail in terms of quality of life. The PSQI has been translated into more than 52 different languages, including the present Arabic version [32, 41].

\section{Statistical Analysis}

Statistical analyses were conducted using Stata version 10.1 (Stata Corporation, College Station, Tex., USA). Differences between groups (study and control) were analyzed using Pearson's $\chi^{2}$ tests (or Fisher's exact tests for cells less than 5). The Student $t$ test was used for comparisons between groups. Comparisons between multiple groups were analyzed using one-way analysis of variance (ANOVA). Post hoc comparisons using Tukey-Kramer tests were used to examine significant differences between groups.

\section{Results}

\section{Comparison of Clinical Features between Groups}

Table 1 summarizes the principal demographic features of the MID patients and controls. The mean ages of the two groups were not significantly different (MID patients: 71 years; controls: 69 years). Sixteen of the patients with MID and 17 subjects in the control group were male, while 4 patients with MID and 1 subject in the control group were female. The proportions of males and females in each diagnostic group were not significantly different. There were no significant differences between the MID group and the control group in years of education. The mean MMSE scores were different between the groups, with the patients with MID scoring lower than the control group (MID patients: 23.5; controls: 28.2; p < 0.05). 
Table 2. Performance of the MID and the control group in the various neuropsychological assessment measures

\begin{tabular}{llrrrr}
\hline & Assessment measure & MID patients & Controls & p value & Post hoc comparison \\
\hline Nonverbal reasoning ability & RPM & $47.1 \pm 8.4$ & $50.7 \pm 7.7$ & 0.180 & control = MID \\
\hline Working memory & digit span forward & $7.5 \pm 1.8$ & $7.7 \pm 1.2$ & 0.736 & control = MID \\
& digit span backward & $5.4 \pm 1.6$ & $6.2 \pm 1.3$ & 0.108 & control = MID \\
& BSRT & $15.8 \pm 3.2$ & $19.5 \pm 2.7$ & $<0.001$ & control $<$ MID \\
& verbal fluency test & $13.7 \pm 4.7$ & $14.7 \pm 2.7$ & 0.440 & control = MID \\
\hline Executive functioning & ToL test & $170 \pm 77$ & $94 \pm 10$ & $<0.001$ & $\begin{array}{l}\text { control }>\text { MID } \\
\text { control }<\text { MID } \\
\end{array}$ \\
& WCST & $2.3 \pm 1.9$ & $3.8 \pm 3.6$ & $<0.001$ & control > MID \\
\hline Affective range & Trail-Making Test & $18.9 \pm 5.0$ & $11.2 \pm 4.5$ & $<0.001$ & control < MID \\
\hline Quality of sleep & GDS & $7.80 \pm 2.46$ & $11.00 \pm 1.24$ & $<0.001$ & control > MID \\
\hline
\end{tabular}

Values are displayed as means \pm SD unless specified otherwise. The $\mathrm{p}$ values are from the one-way ANOVA comparing the groups.

\section{Comparison of Performance between Groups in the Index of Intellectual Functioning}

One-way ANOVA showed no significant difference between the patients with MID and the control group in the presently operationalized index of intellectual functioning, i.e. the RPM $(\mathrm{p}=0.180)$. Post hoc comparisons indicated that the RPM score was not significantly different between the MID and the control group (table 2).

\section{Comparison of Performance between Groups in Indices of Working Memory}

The digit span test, the BSRT and the verbal fluency test appear to tax one's integrity of short-term memory; recall and fluency were operationalized here to gauge working memory. There were no significant group effects on the digit span test (forward and backward; $p=$ 0.736). Post hoc analyses showed that there was no significant difference between the MID patients and the controls. In the verbal fluency test, as well, there appeared to be no difference in performance between the MID and control groups $(\mathrm{p}=0.440)$. Only the BSRT showed a significant difference between the two groups, with the MID patients outperforming the control group.

\section{Comparison of Performance between Groups in Indices of Executive Functioning}

Three measures were operationalized here as executive functioning, namely, the ToL test, the WCST and the Trail-Making Test. There was a significant effect between groups in all 3 psychometric measures $(p<0.001)$. Post hoc comparisons showed that the MID patients were impaired on each of these measures. Overall, the patients with MID performed more poorly than the age-matched controls.

\section{Comparison of Performance between Groups in the Index of Affective Range}

There was a significant group effect on the GDS, a measure of affective functioning ( $\mathrm{p}<$ 0.001 ). Post hoc analyses showed that the patients with MID were significantly impaired as compared with the control group. 


\section{Quality of Sleep}

There was a significant difference between the MID and the control group in quality of sleep as indicated by the PSQI $(p<0.001)$. The patients with MID had significantly higher scores on the PSQI than the controls. Post hoc analyses showed that the patients with MID had a significantly poorer quality of sleep than the control group.

\section{Discussion}

This study employed several measures to assess performance in neurocognitive tests, affective range and quality of sleep in a group of patients with MID presenting at an outpatient clinic in Oman as well as in a group of age- and gender-matched individuals. To our knowledge, this is the first preliminary study that used these indices in an Arab/Islamic population. Most of the discussion revolves around the fact that some of the developing countries have a 'youth bulge' and that, therefore, these societies are spared the vagaries of cognitive impairment. Notwithstanding such a view, a country such as Oman has a population with all the essential features that could trigger cognitive impairment. A recent epidemiological study has indicated that hypertension, hyperlipidemia, diabetes and inflammatory diseases, which are the prime culprits in cognitive impairment, are common in Oman [42]. For instance, the effective management of diabetes, an important risk factor for $\mathrm{AD}$, appears to prevent the progression of neurocognitive impairment [43]. However, the low level of awareness and care seeking in the instance of diabetes in Oman is most disheartening [44]. This means that Oman is likely to be gripped by the emergence of factors leading to cognitive impairment. Our study therefore has the potential to lay the groundwork for the identification of individuals with cognitive impairment.

One of the intractable issues with the rising tide of neurocognitive impairment is how to accurately diagnose the presence of a prodromal neuropsychological state so that potentially modifiable risk factors could be identified and so that preventative measures could be contemplated. A difficulty arises when accurately defining what constitutes cognitive impairment [45]. In this study, the assessment of the severity of cognitive impairment was based on performance in the MMSE or Folstein test. Traditionally, impaired short-term memory among individuals with MCI has been thought to represent prodromal-state dementia. There is increasing recognition that amnestic features are not a prodromal neuropsychological state. In this study, amnestic features were gauged with measures sensitive to working memory, using the digit span test, the verbal fluency test and the BSRT. Consistent with the emerging literature, rather than with the literature on short-term memory, individuals with MCI do not perform significantly differently from healthy subjects. However, differently from individuals with amnestic MCI, it appears that the patients with MID in the tertiary care facility in Oman performed significantly more poorly than the control group on the indices of executive functioning.

Executive functioning is a generic term for various interrelated cognitive functions associated with planning for the future, judgment, decision-making skills, attention span and inhibition. In general, it has been suggested that executive functioning is crucially involved in the sequential organization of behavioral responses to changing environmental cues [46]. In traditional neuropsychology, executive functioning - or its pathological part executive dysfunction - mimics what was once considered a frontal lobe syndrome. The frontal lobe has been associated with diverse functions. Damage to the frontal lobe can occur in several ways including by transient ischemic attacks and/or stroke leading to a number of different dysexecutive symptoms. A dysfunction of this system could lead to impulsive disorganized behavior or simply a poor capacity for planning $[47,48]$. Reflecting the consensus that critical roles for 
the frontal cortex are strategy formation and response monitoring, the terms 'executive function' and 'dysexecutive syndrome' have been coined, respectively, to describe the functions of the intact frontal cortex and the deficits resulting when it is damaged [49]. Regions which are crucially involved in executive functioning are likely to be highly vascularized and, therefore, vulnerable to microvascular damage, which, in turn, might present as MID. In support of the present study, executive dysfunction has been found to be common in individuals with MCI that is associated with MID [50]. More studies are therefore needed to delineate whether MID is more often marked by executive dysfunction than by amnestic features. While this is pending, the present study has shown that executive dysfunctions are early phenomena in individuals with MID.

Our study also employed the GDS to screen for the presence of depression as a measure of affective range among subjects with MID compared with healthy subjects. Numerous studies have shown that depression is prevalent among patients with AD and MID, and that dementia represents a risk factor for depression in the elderly [51, 52]. However, depression is often underdiagnosed in dementia patients due to overlapping motor deficits and cognitive impairment [53]. For instance, loss of interest and apathy are routinely mistaken for symptoms of psychomotor retardation rather than depression. Our data show that depression is more prevalent among individuals with MID than among age-matched controls. Overall, this study provides preliminary empirical evidence that mood dysfunction is detectable in subjects with MID. It challenges the previous view that people in non-Western populations such as those in Oman are unlikely to endorse afflictive emotions [54]. This was based on the assumption that cultural teachings in Oman promoted an odium for distress that had a direct bearing on somatopsychic rather than psychological symptoms. It is worthwhile to note that the GDS is not equipped to elicit the presence of somatic symptoms of depression [55].

Additionally, another study has shown that cognitive deficits are often associated with disrupted sleep patterns, which consequently amplify cognitive impairment [56]. However, there is a paucity of studies examining the quality of sleep in Arab/Islamic countries. For this reason, we investigated the quality of sleep in MID patients versus control patients using the PSQI. Our study showed that the patients with MID had evident sleep disturbances and higher PSQI scores than the controls. It verifies the results of previous work which showed that disordered sleep may occur even in the earliest stages of MID [57]. The presence of disturbed sleep, impaired sleep-wake cycles and other disruptions in circadian rhythms are unfortunate, since the quality of sleep has been shown to be an important element underlying the integrity of normal cognitive functioning [58]. These sleep disturbances are likely to occur in response to vascular damage which may disrupt neural circuits governing sleep control, and/ or a central imbalance in the levels of the important neurotransmitters serotonin, melatonin and acetylcholine, leading to the formation of sleep structural abnormalities.

Some obvious limitations of this study ought to be highlighted. Firstly, the present cohort is of an 'opportunistic' type. In Oman, individuals with personal distress that does not constitute an overt physical illness often do not seek medical consultation. Many types of difficulties are often handled by culture-specific healing systems or are simply perceived as fate $[59,60]$. As the subjects were derived from an urban population and a clinical setting rather than the whole community, any generalization of the results of the present study should be made with caution. In Oman, as in other Eastern societies, the elderly are treated with unquestionable high respect, and, therefore, occasional lapses of memory and disinhibition are likely to be tolerated and are unlikely to reach the modern health care setting. Secondly, in the present study, we took the liberty of demarcating different cognitive domains as different entities. For example, the RPM was operationalized to be sensitive for the nonverbal testing of intellectual ability. However, in the literature, the RPM has been suggested to be subserving the measurement of executive functioning [61]. Similarly, there is likely to be an overlap 
between what is presently operationalized as working memory and what is operationalized as executive functioning $[28,62]$. Thirdly, although most of the measures were nonverbal, it is not clear how 'culture free' these measures are. For this reason, a comparison was made with the healthy subjects in order to elucidate how the MID patients 'deviated' from them. Similarly, some measures were verbal, such as the GDS and PSQI. Although, as mentioned earlier, the psychometric properties of these measures have been established among Arabicspeaking populations, some conceptual misunderstanding could hamper the applicability of such measures to cross-cultural populations. It is therefore imperative that a concerted effort be made in the future to standardize these measures.

\section{Conclusions}

This study embarked on comparing the performance of subjects with MID and their healthy counterparts matched for specific sociodemographic backgrounds. Three interrelated domains were explored: reasoning ability, working memory and executive functioning. The presence of affective disorder and the quality of sleep were also explored. Compared with the healthy subjects, the individuals with MID performed differently in the indices of executive functioning. In particular, the underlying executive dysfunction appears to represent a core cognitive deficit. While a definitive diagnosis of MID is fraught with difficulty in the early stages of the disease, this study should be considered standing sentinel over the prevailing situation in the Arab region and laying some groundwork for further research.

\section{Acknowledgements}

We would like to thank the Sultan Qaboos University Hospital staff and the participants for their cooperation during this study. This project was supported by a research grant from the Research Council, Oman (RC/AGR/FOOD/11/01). Nady Braidy is the recipient of an Alzheimer's Australia Viertel Foundation Postdoctoral Research Fellowship and an NHMRC Early Career Postdoctoral Research Fellowship at the University of New South Wales.

\section{Disclosure Statement}

The authors declare no conflicts of interest.

\section{References}

1 Jiang T, et al: Epidemiology and etiology of Alzheimer's disease: from genetic to non-genetic factors. Curr Alzheimer Res 2013;10:852-867.

-2 Imfeld P, et al: Epidemiology, co-morbidities, and medication use of patients with Alzheimer's disease or vascular dementia in the UK. J Alzheimers Dis 2013;35:565-573.

-3 Israeli-Korn SD, et al: Hypertension increases the probability of Alzheimer's disease and of mild cognitive impairment in an Arab community in northern Israel. Neuroepidemiology 2010;34:99-105.

4 Adeghate E, Donath T, Adem A: Alzheimer disease and diabetes mellitus: do they have anything in common? Curr Alzheimer Res 2013;10:609-617.

5 Catindig JA, et al: Epidemiology of dementia in Asia: insights on prevalence, trends and novel risk factors. J Neurol Sci 2012;321:11-16.

6 Shelley BP, Al Khabouri J: The spectrum of dementia: frequency, causes and clinical profile. A national referral hospital-based study in Oman. Dement Geriatr Cogn Disord 2007;24:280-287. 
-7 Howler E: Biography and dementia. Origin of challenging behavior in patients with multi-infarct dementia or senile dementia of the Alzheimer type in long-term care with reference to the biographical level (in German). Pflege Z 2011;64:612-615.

-8 Zhang X, et al: Effects of acupuncture on declined cerebral blood flow, impaired mitochondrial respiratory function and oxidative stress in multi-infarct dementia rats. Neurochem Int 2013;65C:23-29.

-9 Busl KM, et al: Prestroke dementia is associated with poor outcomes after reperfusion therapy among elderly stroke patients. J Stroke Cerebrovasc Dis 2013;22:718-724.

10 Allan LM, et al: Long term incidence of dementia, predictors of mortality and pathological diagnosis in older stroke survivors. Brain 2011;134:3716-3727.

11 Firbank MJ, et al: Neuroimaging predictors of death and dementia in a cohort of older stroke survivors. J Neurol Neurosurg Psychiatry 2012;83:263-267.

12 Cumming TB, Brodtmann A: Can stroke cause neurodegenerative dementia? Int J Stroke 2011;6:416-424.

13 Vascular health vital to optimal heart and brain function. Treating hypertension and atherosclerosis may help prevent stroke and dementia. Heart Advis 2011;14:1, 11.

14 Gorelick PB, et al: Vascular contributions to cognitive impairment and dementia: a statement for healthcare professionals from the American Heart Association/American Stroke Association. Stroke 2011;42:26722713.

15 Narasimhalu K, et al: The prognostic effects of poststroke cognitive impairment no dementia and domainspecific cognitive impairments in nondisabled ischemic stroke patients. Stroke 2011;42:883-888.

16 Tanabe J: White matter hyperintensities are associated with an increased risk of stroke, dementia and mortality. Evid Based Ment Health 2011;14:1.

17 Tang WK, et al: Absence of cerebral microbleeds predicts reversion of vascular 'cognitive impairment no dementia' in stroke. Int J Stroke 2011;6:498-505.

18 Alvarez-Sabin J, Roman GC: Citicoline in vascular cognitive impairment and vascular dementia after stroke. Stroke 2011;42(suppl):S40-S43.

19 Al-Adawi S: Emergence of diseases of affluence in Oman: where do they feature in the health research agenda? Sultan Qaboos Univ Med J 2006;6:3-9.

20 Al-Sinawi $\mathrm{H}$, et al: Emerging burden of frail young and elderly persons in Oman: for whom the bell tolls? Sultan Qaboos Univ Med J 2012;12:169-176.

-21 Bowirrat A, Oscar-Berman M, Logroscino G: Association of depression with Alzheimer's disease and vascular dementia in an elderly Arab population of Wadi-Ara, Israel. Int J Geriatr Psychiatry 2006;21:246-251.

-22 Bowirrat A, et al: Prevalence of Alzheimer's type dementia in an elderly Arab population. Eur J Neurol 2001; 8:119-123.

23 Zhu B, et al: The SEMA5A gene is associated with hippocampal volume, and their interaction is associated with performance on Raven's Progressive Matrices. Neuroimage 2013;88C:181-187.

24 Fajgelj S, Bala G, Katic R: Latent structure of Raven's Colored Progressive Matrices. Coll Antropol 2010;34: 1015-1026.

-25 Spencer RJ, et al: WAIS-IV reliable digit span is no more accurate than age-corrected scaled score as an indicator of invalid performance in a veteran sample undergoing evaluation for mTBI. Clin Neuropsychol 2013; 27:1362-1372.

-26 Massuda R, et al: Verbal memory impairment in healthy siblings of patients with schizophrenia. Schizophr Res 2013;150:580-582.

27 Buschke H, Fuld PA: Evaluating storage, retention, and retrieval in disordered memory and learning. Neurology 1974;24:1019-1025.

28 Al-Adawi S, Powell JH, Greenwood RJ: Motivational deficits after brain injury: a neuropsychological approach using new assessment techniques. Neuropsychology 1998;12:115-124.

29 Rosen V, et al: Working memory and apolipoprotein E: what's the connection? Neuropsychologia 2002;40: 2226-2233.

-30 Cowan N: What are the differences between long-term, short-term, and working memory? Prog Brain Res 2008;169:323-338.

-31 Moscovitch M: Memory and working-with-memory: a component process model based on modules and central systems. J Cogn Neurosci 1992;4:257-267.

-32 Al-Adawi S, et al: Neurocognitive functioning in women presenting with undifferentiated somatoform disorders in Oman. Psychiatry Clin Neurosci 2010;64:555-564.

33 Benton AL: Differential behavioral effects in frontal lobe disease. Neuropsychologia 1968;6:53-60.

-34 Sciarma T, et al: The verbal fluency test for the diagnosis of dementia (in Italian). Riv Neurol 1990;60:194-197.

Nishimura Y, et al: Association of decreased prefrontal hemodynamic response during a verbal fluency task with EGR3 gene polymorphism in patients with schizophrenia and in healthy individuals. Neuroimage 2014; 85:527-534.

-36 Krueger CE, et al: Double dissociation in the anatomy of socioemotional disinhibition and executive functioning in dementia. Neuropsychology 2011;25:249-259.

37 Nelson HE: A modified card sorting test sensitive to frontal lobe defects. Cortex 1976;12:313-324.

-38 Yesavage JA, et al: Development and validation of a geriatric depression screening scale: a preliminary report. J Psychiatr Res 1982;17:37-49. 
-39 Conradsson M, et al: Usefulness of the Geriatric Depression Scale 15-item version among very old people with and without cognitive impairment. Aging Ment Health 2013;17:638-645.

40 Chaaya M, et al: Validation of the Arabic version of the short Geriatric Depression Scale (GDS-15). Int Psychogeriatr 2008;20:571-581.

41 Suleiman KH, et al: Translating the Pittsburgh Sleep Quality Index into Arabic. West J Nurs Res 2010;32:250268.

42 Mokdad AH, et al: The state of health in the Arab world, 1990-2010: an analysis of the burden of diseases, injuries, and risk factors. Lancet 2014;383:309-320.

43 Dominguez R0, et al: Type 2 diabetes and/or its treatment leads to less cognitive impairment in Alzheimer's disease patients. Diabetes Res Clin Pract 2012;98:68-74.

44 Elliott JA, et al: Diabetes self-management and education of people living with diabetes: a survey in primary health care in Muscat Oman. PLoS One 2013;8:e57400.

45 Ghosh S, Libon D, Lippa C: Mild cognitive impairment: a brief review and suggested clinical algorithm. Am J Alzheimers Dis Other Demen 2013, Epub ahead of print.

46 Falkowski J, et al: Executive functioning and the metabolic syndrome: a project FRONTIER study. Arch Clin Neuropsychol 2014;29:47-53.

47 Longo CA, Kerr EN, Smith ML: Executive functioning in children with intractable frontal lobe or temporal lobe epilepsy. Epilepsy Behav 2013;26:102-108.

48 Dulay MF, et al: Executive functioning and depressed mood before and after unilateral frontal lobe resection for intractable epilepsy. Neuropsychologia 2013;51:1370-1376.

49 Baddeley A, Wilson B: Frontal amnesia and the dysexecutive syndrome. Brain Cogn 1988;7:212-230.

50 Jia J, et al: The prevalence of mild cognitive impairment and its etiological subtypes in elderly Chinese. Alzheimers Dement 2014;10:439-447.

51 Chen PJ, et al: The temporal relationship between depressive symptoms and dementia: a community-based prospective study. Arch Gen Psychiatry 1999;56:261-266.

52 Richard E, et al: Late-life depression, mild cognitive impairment, and dementia. JAMA Neurol 2013;70:383389.

53 Downing LJ, Caprio TV, Lyness JM: Geriatric psychiatry review: differential diagnosis and treatment of the 3 D’s - delirium, dementia, and depression. Curr Psychiatry Rep 2013;15:365.

54 Eloul L, Ambusaidi A, Al-Adawi S: Silent epidemic of depression in women in the Middle East and North Africa region: emerging tribulation or fallacy? Sultan Qaboos Univ Med J 2009;9:5-15.

55 Mitchell AJ, et al: Diagnostic validity and added value of the Geriatric Depression Scale for depression in primary care: a meta-analysis of GDS30 and GDS15. J Affect Disord 2010;125:10-17.

56 Al-Adawi S, Burke DT, Dorvlo ASS: The effect of methylphenidate on the sleep-wake cycle of brain-injured patients undergoing rehabilitation. Sleep Med 2006;7:287-291.

57 Naismith SL, et al: Circadian misalignment and sleep disruption in mild cognitive impairment. J Alzheimers Dis 2014;38:857-866.

58 Roh JH, et al: Disruption of the sleep-wake cycle and diurnal fluctuation of $\beta$-amyloid in mice with Alzheimer's disease pathology. Sci Transl Med 2012;4:150ra122.

$\checkmark 59$ Al Asmi A, et al: Types and sociodemographic correlates of complementary and alternative medicine (CAM) use among people with epilepsy in Oman. Epilepsy Behav 2013;29:361-366.

60 Al-Adawi S, et al: Psychosocial aspects of epilepsy in Oman: attitude of health personnel. Epilepsia 2001;42: 1476-1481.

61 Perfetti B, et al: Differential patterns of cortical activation as a function of fluid reasoning complexity. Hum Brain Mapp 2009;30:497-510.

62 Roussel M, et al: Is the frontal dysexecutive syndrome due to a working memory deficit? Evidence from patients with stroke. Brain 2012;135:2192-2201. 


\section{Erratum}

In the article by Al-Adawi S, Braidy N, Essa M, Al-Azri F, Hussain S, Al-Sibani N, Al-Khabouri J, Al-Asmi A, Al-Mashani A, entitled 'Cognitive profiles in patients with multi-infarct dementia: an Omani study' [Dement Geriatr Cogn Disord Extra 2014;4:271-282, DOI:10.1159/000363621, the author Essa M. was spelled wrongly. It should read Essa MM. 